\title{
Research of Physical Experiment Teaching and Quality Reform Jing $\mathrm{Di}^{1 \text {, a }}$ \\ ${ }^{1}$ Department of Physical Education, Yulin University, Yulin, Shaanxi, 719000 \\ ${ }^{a}$ email
}

Keywords: Physical Experiment, Teaching and Quality, Reform

\begin{abstract}
Physical Experiment teaching is an important part of the whole process of teaching PE Colleges. To make students to have basic high-quality, comprehensive ability, spirit of innovation, only depends on teaching classes and theoretical study is difficult to meet the requirements. In this capacity formation process, experimental teaching plays an important role. It not only validates classroom theory, but also explores new areas of knowledge. Experimental teaching is the main way to train students to integrate theory with practice, increase ability and comprehensive practical ability. It can not only help students digest, absorb, consolidate the theoretical knowledge they learned, but can develop rigorous scientific attitude, scientific laboratory capacity and the ability to use integrated discipline theory to analyze problems.
\end{abstract}

\section{Introduction}

Sports Experiment Lessons is an important part of the Physical Education Basic Education Course, experimental teaching in quality education, especially in the culture of innovation awareness, training students to identify problems, ask questions and problem solving practice ability unique role, which any other teaching can not be replaced. However, previous experiments by sports disciplines into motion anatomy experiment, exercise physiology experiments, sports health science experiments, sports surveying experiment, exercise biochemistry (mechanics) experiments. Many institutions have a larger proportion of the experimental class sports anatomy, exercise physiology, sports biochemistry, physical health care, respectively, according to discipline up labs, belonging to the Department of leadership theory in management.

In the experimental teaching, through the division of labor, so that students understand the importance of the relationship between man and man, the individual and the collective of experimental and scientific research, students of unity and cooperation can develop collective spirit of collaboration; through standardized operation, strict requirements, students can develop realistic scientific attitude and rigorous and realistic style of study; through debugging equipment, troubleshooting, as well as failures and setbacks, can cultivate the spirit of exploration and strong willpower students diligent study, while students' practical point of view, to understand the practice is to recognize source, is the sole criterion for testing truth, to look beyond the surface, comprehensive and objective look at the problem dialectical materialist world outlook; experimental phenomena through observation, analysis of experimental results, students can develop a keen intuition, creative thinking and innovation spirit; through installation, commissioning, maintenance equipment, duty and other arrangements can be cultured from all round view of the concept of student work, love and moral civil matter, the overall quality of disciplined self-cultivation, and good hygiene practices. Physical Experiment teaching is an important and indispensable part of the whole process of teaching professional sports. However, for various reasons, we have been unable to obtain experimental teaching PE enough attention, let alone improve and the students at the school only pay attention to professional and technical courses and theory courses while ignoring the experimental class. This runs counter to the need to train new and innovative talents of our educational goals. Of course, this experiment closely and Present PE Teaching. 


\section{Physical Experiment Teaching and Quality Problems}

Over the years, sports has been teaching experimental subordination theory of teaching, still followed the traditional mode of education, Sports Experiment Lessons failed to make breakthroughs in the theoretical teaching system, not according to their own systematic experimental techniques and scientific teaching, the lack of a separate Let classes, systemic difference. In theory focuses on educational concepts exist, the tendency of light practice, theory and experiment courses Course unreasonable proportion, does not reflect their own teaching practice and systemic coherence. Experiment Course theory courses are often run pilot projects to be arbitrarily changed.

For example, in exercise physiology experiments in existing blood pressure measuring pulse and body composition was measured in experiments with anthropometric evaluation or recurring Sport Health, wasteful experiment content, class and talent matter. Main courses experimental teaching and experimental task is to verify the theory of culture experiments skills. Resulting in excessive classic experiment, reflecting little experiment of modern science and technology development; excessive confirmatory test, and training creative ability of applications, integrated, design of experiments is too small, but the lack of obstacles to experiment . Especially experimental teaching and social services, scientific research and production of the first line out of touch with today's high-tech far apart, lack of sense of the times. In the experimental teaching process should not only tell the student pilot project, as well as detailed experimental principle, the list of equipment materials, experimental procedures, data recording forms and the precautions to be, as long as students copy or imitate, according to side fill a prescription to complete the experimental task such holding down teaching model is difficult to play the initiative of students. Students at the school can not obtain experimental techniques and improve the theoretical system of experimental skills training, reducing the knowledge of migration, to take the initiative of hindered. The rise of the knowledge economy today, just legalistic learning old knowledge, is not adapt to the development of society, let alone implement innovative education and individualized education.

Students in class arbitrary grouping is not clear, the instrument damage compensation system is not strict, or even impossible to implement. Assessment and quality control mechanism is not perfect, the experimental class students' enthusiasm is not high, the initiative is not strong.

\section{Physical Experiment Teaching Reform}

Rescreening, optimization, reorganization of teaching content has reform of experimental teaching curriculum. Course content is a means to achieve program goals, specific curriculum objectives to be translated into specific knowledge and skills [6]. In the experimental options educational goals, lesson objectives set principle of unity, reform the existing experimental teaching programs and content based on a comprehensive, practical, and scientific point of principle, delete and update does not comply with the direction of development of modern science content compression section classic but behind the content, reduce duplication of validation experiments, the addition of some applications and designed, comprehensive, consistent with the requirements of the new situation of the project, namely the content of the experimental attention-looking, scientific and reasonable.

In the experimental teaching process, teaching content arrangements should be based on teaching plans as the basis, taking full account of their level of knowledge and cognitive features, based on the teaching content in accordance with the degree of difficulty, from simple to complex sequence reasonable arrangements, namely, the basic theoretical basis for experimental y y y comprehensive experiment design experiment. So that students master the basic experimental methods and skills in a series of experimental activities and to gradually increase the ability and the ability of the integrated use of knowledge students develop their ability to analyze and solve problems, and improve students' sense of innovation, enhance the experiment Lesson learning interest and confidence.

To carry out students' comprehensive ability to use knowledge and practical ability, experimental 
design, innovation-based integrated in higher grades and design of experiments. Comprehensive experiment based, and the proportion of hours designing experiment by $3 / 5,1 / 5$, respectively, to take independent modes of teaching, it is recommended in the fifth or sixth semester. Comprehensive experimental projects are such as the comprehensive evaluation of the exercise load and the like.

Design of experiments the students own access to relevant information, with reference to the design requirements of the experiment, the experimental conditions of teachers given the purpose of the experiment, the experimental program, but also can design their own experiments, reflecting inquiry can give students sufficient space and room. Choose the design of the experiment should not be too complicated, only emphasized the design and distinctive. Choose sports disciplines representative and universal means more advanced method, test targets relatively diverse equipment as the primary means of experiments can be used to currently available or will be purchased by large modern advanced equipment (such as exercise treadmill, semi-automatic biochemical analyzer, body composition analyzer, etc.). Throughout the design of the experiment, so that students will always be in active status, the use of multi-disciplinary, multi-effect indicators, comprehensive analysis, to solve one or two problems, the initial grasp of some commonly used methods of sports human science experiment. The main types of experiments at a higher level to improve students' comprehensive ability to use knowledge and to explore the issues and to analyze and solve scientific problems thinking, students of originality, creative thinking work, novelty and so on.

The use of modern technology and advanced experimental teaching methods are important. Reform the traditional teaching model, to strengthen the high-tech applications in the experimental teaching, and gradually realize the modernization of the experimental teaching methods. For example, electronic teaching, multimedia teaching, simulation technology, computer network teaching, satellite TV network technology teaching methods used in experimental teaching, and online virtual lab appears, not only can make the experiment content has increased significantly shorten test time, save a lot of manpower, material and financial resources, and the experiment vivid, clear, help to deepen understanding of the principles of the experiment. Its intuitive operability, knowledge, and interest earned student's interest and love, but also cultivate students' ability to use modern technology.

Laboratory means a period of time after the end of the experiment course provides students with a place to practice in order to consolidate the experimental skills. In sports experiment, a considerable part is to master technology and techniques as the core content, such as massage, bandaging, cardiopulmonary resuscitation and the like. This section does not through long-term practice, practice makes perfect can not be achieved, to apply their knowledge of the purpose. Therefore, open laboratory methods may be used. So that students can appreciate the value of experimental skills, thereby increasing interest in learning to play its learning initiative.

Teachers directly involved in research sub-topics by selecting a portion of outstanding students, and other research projects, teachers in the research work should be possible to absorb the students, for students through a more thorough understanding of the research process and a deeper understanding of scientific phenomena, exercise the necessary scientific research ability. Meanwhile, with the continuous improvement of the sports lab laboratory conditions gradually expanded to areas of social service. For example, assume an outer part of the unit sports a sister unit test and assume experimental teaching and research, and further develop their innovative spirit and practical ability and scientific research ability.

Textbook construction is an important part of the subject construction and development, which is particularly important for an emerging discipline is concerned. Currently, teaching material construction of sport and PE college human function experiment course still very backward. Thus, according to the training objectives and the Ministry of Education on Physical Experiment Teaching Guidelines content requirements, in full consultation with experts in various disciplines opinions basis, through content integration and restructuring of sports experiment course, the absorption current advanced experimental and technological achievements prepare to adapt to the sports professional education programs and curricula, determine the content and structure of the system of 
professional sports experiment teaching materials, and as a basis for the preparation of the corresponding experimental materials. For acceleration of Sports College teaching reform pace, the creation of a complete experimental teaching system plays an important role.

Experimental examination is to test the effect of experiment teaching, improve teaching quality of experimental protection. Both teachers understand the effect of experimental teaching, but also test students during the experiment demonstrated thinking, qualities and abilities.

\section{Conclusion}

Physical experiment teaching has an irreplaceable role in physical training under the new century. Under the status of PE experiment teaching challenges of the 21st century and the new educational reform goals, deepen the teaching reform experiment is trend, the only way is to improve the quality of education. We think this paper should be meaningful for the PE experimental teaching reform.

\section{References}

[1] Meng Fangang. Experiment on the reform of teaching methods [J]. Laboratory Science. 2000, (1).

[2] Liu Zhen. The Role of Modern Educational Technology in Quality Education at Teachers College of [J]. Educational research. 2000, (9).

[3] Cai Xinsheng, Basic Experience of College Experiment Teaching Reform [J]. Laboratory Research and Exploration. 2001, (1): 1, 2.

[4] Xu Jianfei. Experimental teaching system reform to strengthen the innovation and personnel training [J]. Laboratory Research and Exploration. 2001, (1): 3) 4.

[5] Liu Liyan. Physical Education Reform experimental design and even practice [J]. Journal of Dalian University. 2000, (3). 